\title{
Measuring crowding: I know it when I see it
}

\author{
Howard Ovens $s^{1,2}\left[\right.$. Alan Drummond ${ }^{3,4,5}$
}

Received: 10 November 2021 / Accepted: 18 November 2021

(C) The Author(s), under exclusive licence to Canadian Association of Emergency Physicians (CAEP)/ Association Canadienne de Médecine d'Urgence (ACMU) 2021

Emergency department (ED) crowding is an all too frequent fact of life for most of us. It is a major factor in shortening the career spans of physicians and nurses, causes untold patient misery, and is a source of heartburn for hospital administrators and politicians. If we had a simple, agreedupon gold standard for a measure of crowding, one that correlated with patient experience and safety, would not that make life better! A single number could tell us whether crowding improved or worsened over time, help us respond to a surge needing additional resources, help us advocate for support and systemic changes, and make research on ED operations and quality improvement easier to do and explain. Where are we in the development of such a measure?

A search on Google Scholar of "emergency department overcrowding" and "measures" or "measurement" or "scale" yields 33,400 results. This edition of CJEM adds two more. Clouston et al. [1] took a modest amount of data from one ED and looked for simple measures of crowding that correlated best with their chosen reference standard: physician perception of crowding. They found "ED occupancy"-ED census divided by the number of staffed beds-showed a strong correlation with physician perception of crowding and either matched or outperformed more complex crowding scores. McRae et al. [2] took a huge database of nearly one million visits to a city's three major EDs and looked for crowding metrics that best predicted the risk of 72-h

\section{Howard Ovens}

howard.ovens@utoronto.ca

1 Schwartz/Reisman Emergency Medicine Institute, Mount Sinai Hospital, Toronto, ON, Canada

2 Department of Family and Community Medicine, University of Toronto, Toronto, ON, Canada

3 Great War Memorial Hospital, Perth, ON, Canada

4 Department of Family Medicine, University of Ottawa, Ottawa, ON, Canada

5 Department of Family Medicine, Queen's University, Kingston, ON, Canada return visits. They grouped their metrics as indicative of input, throughput, and output and identified the most predictive metric from each area; they found ED waiting time, ED occupancy, and boarding times performed the best, although the effect sizes seem modest to us.

What are we to make of these results? Each paper attempts to add clarity to an important and vexing problem. Both produced results that make some intuitive sense, but both are limited by the lack of a pre-existing gold standard against which to measure their results. The McRae investigators chose 72-h revisit rates as their outcome of interest and worked backward to find metrics that predicted an increase. At least the Clouston team's metric of ED occupancy came out on top as the throughput indicator of choice in the McRae study, lending it some external validation. Both papers are urban and academic centric, and their relevance to medium and smaller community EDs is uncertain.

When we talk about ED crowding, we must keep in mind the many different stakeholders and their perspectives. Patients are our most important stakeholder group, yet ED crowding, waiting, and service may be experienced quite differently by newly arriving patients, those in process, or those waiting for a bed. Even within the arriving patient cohort, wait times - and perceptions of wait times - might vary considerably for certain patient cohorts, such as those with mental illness or addiction. And paramedics, consulting staff, and emergency staff might also have very different perspectives..

The metrics and the language we use to describe crowding must be relevant to the intended purpose; a single metric cannot meet all our needs. The CAEP Position Statement on Overcrowding [3] suggested five measures of ED waiting and care as the minimum data set for describing ED operations and degree of crowding. They included two measures of pure waiting (time to initial physician assessment and time from order to admit to bed transfer) as well as three measures of care time (length of stay in the ED in cohorts of admitted patients, CTAS I-III discharged patients, and CTAS IV/V discharged patients). We have tracked those 
five indicators in Ontario since 2008, adding ambulance offload time several years ago; given the resource implications and impact on community safety, it seems ambulance offload time is another pure wait time worth tracking. Taken together, they have been very useful in comparing a province's EDs to each other and the system's overall performance over time. In many hospitals, performance in a single indicator has guided the need to conduct a quality improvement effort targeted to the area that is underperforming-from ambulance offload processes at the front end of the ED to hospital processes to reduce boarding times. At the hospital and system levels, these six indicators have stood the test of time and are easily explained and understood.

From the two papers, the one finding that may have an impact on our work on crowding is the simplicity and utility of ED occupancy as a descriptor of an ED at a point in time. It not only reflects the size of an ED and the number of patients under care, it is also sensitive to staffing levels.

In spring 2020 the COVID-19 pandemic created a shortterm, system-wide relief from crowding. ED staff cancelled their holidays and showed up to work in droves; hospital operations shifted and admitted bed flow became almost universally superb; and our usual patient volumes dropped by $50 \%$ as people stayed away due to fears of COVID- 19 . Despite the risk of contracting the disease, working conditions were otherwise relatively ideal. Eighteen months later, the situation has changed drastically; hospitals are full, EDs are back to usual volumes but with higher acuity and complexity than ever, and staffing levels are dangerously reduced in many if not most Canadian EDs. Wait times, ambulance offload times, and likely every other metric of relevance are concerning. We have the metrics we need to address almost any need to describe or quantify crowding. What we face is more a political problem than a measurement one: convincing those who can make a difference that crowding can be fixed.

\section{Declarations}

Conflict of interest The authors have no conflicts to declare.

\section{References}

1. Clouston R, Atkinson P, Canales DD, et al. Emergency department occupancy is useful as a simple real-time measure of crowding. CJEM. 2021. https://doi.org/10.1007/s43678-021-00098-8.

2. McRae AD, Rowe BH, Usman I, et al. Finding the most meaningful ED crowding metrics: a comparative evaluation of the strengths of association between different emergency department crowding metrics and repeat visits within 72 hours. CJEM. 2021. https://doi.org/10.1007/s43678-021-00234-4.

3. Affleck A, Parks P, Drummond A, et al. Emergency department overcrowding and access block. CJEM. 2013;15(6):359-70. 